\title{
STRUCTURE THEOREM FOR COMPACT VAISMAN MANIFOLDS
}

\author{
Liviu Ornea And Misha Verbitsky
}

\begin{abstract}
A locally conformally Kähler (l.c.K.) manifold is a complex manifold admitting a Kähler covering $\tilde{M}$, with each deck transformation acting by Kähler homotheties. A compact l.c.K. manifold is Vaisman if it admits a holomorphic flow acting by non-trivial homotheties on $\tilde{M}$. We prove a structure theorem for compact Vaisman manifolds. Every compact Vaisman manifold $M$ is fibered over a circle, the fibers are Sasakian, the fibration is locally trivial, and $M$ is reconstructed from the Sasakian structure on the fibers and the monodromy automorphism induced by this fibration. This construction is canonical and functorial in both directions.
\end{abstract}

\section{Introduction}

Let $(M, J, g)$ be a connected complex manifold of complex dimension $n \geqslant 2$, equipped with a Hermitian metric. We shall denote by $\omega$ its fundamental twoform given by $\omega(X, Y)=g(X, J Y)$.

Definition 1.1: $(M, J, g)$ is called locally conformally Kähler, l.c.K. for short, if there exists an open cover $\mathcal{U}=\left\{U_{\alpha}\right\}$ such that each locally defined metric $g_{\alpha}=e^{-f_{\alpha}} g_{U_{\alpha}}$ is Kähler for some smooth function $f_{\alpha}$ on $U_{\alpha}$.

Equivalently, $(M, J, g)$ is l.c.K. if and only if there exists a closed one-form $\theta$ such that

$$
d \omega=\theta \wedge \omega .
$$

Locally, $\omega_{\mid U}=d f_{U}$. Note also that on complex surfaces, the equation (1.1) implies $d \theta=0$.

The globally defined one-form $\theta$ is called the Lee form and its metrically equivalent (with respect to $g$ ) vector field $v=\theta^{\sharp}$ is called the Lee vector field.

An equivalent definition of l.c.K. manifolds is obtained via the universal Riemannian cover. Let $p: \tilde{M} \rightarrow M$ be the covering map and denote also by $J$ the

Received May 23, 2003.

Liviu Ornea is a member of EDGE, Research Training Network HRPN-CT-2000-00101, supported by the European Human Potential Programme.

Misha Verbitsky is an EPSRC advanced fellow supported by CRDF grant RM1-2354-MO02 and EPSRC grant GR/R77773/01.

2000 Mathematics Subject Classification. 53C55, 53C25.

Key words and phrases. Locally conformal Kähler manifold, Sasakian manifold, parallel Lee form, Gauduchon metric, weight bundle, monodromy. 
lifted complex structure. The lifted metric $\tilde{g}$ is globally conformal with a Kähler metric $g_{k}$, because $p^{*} \theta$ is exact. The fundamental group of $M$ will then act by holomorphic conformal transformations with respect to $(J, \tilde{h})$. But conformal transformations of a symplectic form (in real dimension at least 4) are in fact homotheties (note that this remains true for the deck group of any covering). The converse is also true, namely: $(M, g, J)$ is l.c.K. if and only if the universal Riemannian covering admits a Kähler form $\omega_{k}$ with respect to which $\pi_{1}(M)$ acts by holomorphic homotheties (cf. [DO]).

For later use, let $\mathcal{H}(\tilde{M}, \tilde{h}, J)$ be the above group of holomorphic homotheties and let

$$
\rho: \mathcal{H}(\tilde{M}, \tilde{h}, J) \longrightarrow \mathbb{R}^{>0}
$$

be the homomorphism associating to each homothety its scale factor.

It will be useful in the sequel to regard l.c.K. geometry in the framework of Weyl geometry (cf. e.g. [G2]). Let $L \rightarrow M$ be the weight bundle, i.e. the bundle of scalars of weight 1 associated to the bundle of linear frames of $(M, g)$ by the representation $G L(n, \mathbb{R}) \ni A \mapsto|\operatorname{det} A|^{\frac{1}{n}}$. The Lee form $\theta$ can be viewed as a connection form in $L$. The induced Weyl connection is clearly flat. We shall refer to the holonomy of this connection as to the monodromy of $L$.

A strictly smaller class of l.c.K. manifolds is the one formed by those with parallel (with respect to the Levi Civita connection) Lee form. We call them Vaisman manifolds, as I. Vaisman was the first to study them sytematically (under the name of generalized Hopf manifolds [V2], [V1], a name which later proved to be inappropriate). On such a manifold, the length of the Lee vector field is constant and we shall always assume it is nonzero. Hence, in what follows, we shall normalize and consider that on a Vaisman manifold $|v|=1$.

Note that on a compact l.c.K. manifold, the metric with parallel Lee form, if it exists, is unique up to homothety in its conformal class and coincide with the Gauduchon (standard) metric, [MPPS]. Hence, on a compact Vaisman manifold, we may always assume that we work with a metric with harmonic Lee form of length 1. Moreover, loc. cit., the group $\operatorname{Aut}(M)$ of l.c.K. automorphisms of a compact Vaisman manifold coincides with the isometry group of the Gauduchon metric, thus being a compact Lie group.

To describe examples of Vaisman manifolds we need to recall the notion of Sasakian manifold (see [Bl] and [BG1] for a survey and references on Sasakian geometry). We need first the following

Definition 1.2: Let $(X, g)$ be a Riemannian manifold. A cone over $X$ is a Riemannian manifold $C(X):=\left(X \times \mathbb{R}^{>0}, d t^{2}+t^{2} g\right)$, where $t$ is the parameter on $\mathbb{R}^{>0}$. For any $\lambda \in \mathbb{R}^{>0}$, the map

$$
\tau_{\lambda}: C(X) \longrightarrow C(X), \quad(x, t) \longrightarrow(x, \lambda t)
$$

multiplies the metric by $\lambda^{2}$. 
Definition 1.3: Let $X$ be a Riemannian manifold. A Sasakian structure on $X$ is a complex structure on $C(X)$ satisfying the following:

(i) The metric on $C(X)$ is Kähler.

(ii) The map $\tau_{\lambda}: C(X) \longrightarrow C(X)$ is holomorphic, for all $\lambda \in \mathbb{R}^{>0}$.

Let $X$ be a Sasakian manifold, and $C(X)$ its Kähler cone. Given a number $q \in \mathbb{R}, q>1$, consider an equivalence relation $\sim_{q}$ on $C(X)=X \times \mathbb{R}^{>0}$ generated by $(x, t) \sim(x, q t)$. Since the map $(x, t) \longrightarrow(x, q t)$ mupltiplies the metric by $q^{2}$, the quotient $M=C(X) / \sim_{q}$ is an l.c.K. manifold. Moreover, $M$ is a Vaisman manifold, with the Gauduchon metric provided by an isomorphism $M \cong X \times S^{1}$.

Conversely, it follows from [KO] (see also [GOP], [Ve]) that any Vaisman manifold $(M, g, J)$ with exact Lee form is a cone of a naturally defined Sasakian manifold. Indeed, if $\theta=d f$, since $|\theta|=$ const, $f: M \longrightarrow \mathbb{R}$ is a Riemannian submersion. The Lee flow $\psi_{t}$ is the gradient flow of $f$. Therefore, $\psi_{t}$ is compatible with $f$ in the following sense:

$$
f\left(\psi_{t} x\right)=f(x)+t
$$

As the Lee field is Killing, $\psi_{t}$ is formed by isometries. Therefore, $\psi_{t}$ induces (locally in $M$ ) a trivialization of the fibration $f: M \longrightarrow \mathbb{R}$ identifying its fibers.

Let $X$ be the fiber of $f$, and let $\rho: M \longrightarrow X$ the corresponding trivialization map. The map $\rho$ is well defined everywhere in $M$ if $\psi_{t}$ is defined for all $t$.

The manifold $M$ is now isomorphic to $X \times \mathbb{R}$. Using exponential map, we identify $X \times \mathbb{R}$ with $X \times \mathbb{R}^{>0}$. The cone metric on $M$ is written as $e^{-f} g$ and it is Kähler. By definition, this implies that $X$ is a Sasakian manifold.

More generally, let $\varphi: X \longrightarrow X$ be an automorphism of a Sasakian structure. The map $(x, t) \stackrel{\varphi_{q}}{\longrightarrow}(\varphi(x), q t)$ is compatible with the complex structure and multiplies the metric by $q^{2}$. Therefore, the quotient $M_{\varphi, q}$ of $C(X)$ by the corresponding equivalence relation $\sim_{\varphi, q}$ is called the suspension of $\varphi$ over the circle of length $2 \pi q$ and is a l.c.K. manifold. The following proposition is implied by $[\mathrm{KO}]$ (see also $[\mathrm{GOP}]$ ).

Proposition 1.4: Assume that the Sasakian manifold $X$ is compact, and $M_{\varphi, q}:=$ $C(X) / \sim_{\varphi, q}$ an l.c.K. manifold constructed as above. Then $M_{\varphi, q}$ is a Vaisman manifold.

The most common examples of this kind are the Hopf surfaces with the metrics constructed in [GO], [Bel], and generalized in [KO].

The aim of this paper is to prove the following converse to the above proposition, which is proven for Einstein-Weyl locally conformally Kähler manifolds in $[\mathrm{Ve}]$ :

Structure Theorem: Let $M$ be a compact Vaisman manifold. Then $M$ admits a canonical Riemannian submersion $p: M \longrightarrow S^{1}$ to a circle, and the fibers of $p$ are isomorphic as Riemannian manifolds. Moreover, for all $s \in S^{1}$, 
the manifold $X=p^{-1}(s)$ is equipped with a natural Sasakian structure and a Sasakian sutomorphism $\varphi$, such that $M$ is isomorphic to the Vaisman manifold $M_{\varphi, q}:=C(X) / \sim_{\varphi, q}$ constructed above.

\section{Proof of the structure theorem}

Let $(M, g, J)$ be a compact Vaisman manifold such that $g$ is the Gauduchon metric. The associated Lee form $\theta$ being harmonic, $M$ is foliated by minimal hypersurfaces whose normal trajectories are geodesics.

To obtain a submersion over $S^{1}$ we proceed as follows. Fix a point $x_{0} \in M$. Consider a smooth path $\gamma:[0,1] \longrightarrow M, \gamma(0)=x_{0}, \gamma(1)=x$. Denote by $R(\gamma, x)$ the integral

$$
R(\gamma, x):=\int_{\gamma} \theta
$$

Since $\theta$ is closed, $R(\gamma, x)$ depends only on the homotopy class of $\gamma$.

Let $\gamma_{1}, \ldots, \gamma_{k}$ be the generators of $H^{1}(M, \mathbb{Z})$. Denote by $\alpha_{1}, \ldots \alpha_{k}$ the periods of $\theta$,

$$
\alpha_{i}:=\int_{\gamma_{i}} \theta
$$

Clearly, $R(\gamma, x)$ defines a function

$$
p: M \longrightarrow \mathbb{R} /\left\langle\alpha_{1}, \ldots \alpha_{k}\right\rangle,
$$

where $\left\langle\alpha_{1}, \ldots \alpha_{k}\right\rangle \subset \mathbb{R}$ is the abelian group generated by $\alpha_{1}, \ldots \alpha_{k}$ and

$$
\mathbb{R} /\left\langle\alpha_{1}, \ldots \alpha_{k}\right\rangle
$$

is the quotient group.

If $\left\langle\alpha_{1}, \ldots \alpha_{k}\right\rangle \cong \mathbb{Z}$, then we have the desired submersion over $S^{1}$. The above condition is certainly satisfied if the monodromy of the flat connection associated to $\theta$ in $L$ is $\mathbb{Z}$. Indeed, let $\gamma$ be a closed loop in $M$. By definition of $L$, the monodromy of $L$ along $\gamma$ is equal to $\int_{\gamma} \theta$. If the monodromy of $L$ is isomorphic to $\mathbb{Z}$, then the periods

$$
\alpha_{i}:=\int_{\gamma_{i}} \theta
$$

are proportional (with integer coefficient) to $\alpha_{0} \in \mathbb{R}$. Then the function $p$ : $M \longrightarrow \mathbb{R} /\left\langle\alpha_{1}, \ldots \alpha_{k}\right\rangle$ maps $M$ to $\mathbb{R} / \mathbb{Z} \alpha_{0}=S^{1}$. The differential of $p$ is equal $\theta$. Passing to a covering, we obtain a map $f: \tilde{M} \longrightarrow \mathbb{R}, d f=\theta, \tilde{M} / Z \cong M$. As we have shown above, $\tilde{M}$ is a cone over a Sasakian manifold: $\tilde{M} \cong C(X)$. The monodromy map $\mu: \tilde{M} \longrightarrow \tilde{M}$ preserves the complex structure and the Lee field and multiplies the Kähler form by a constant. Therefore, $\mu$ is induced by a Sasakian automorphism $\varphi: X \longrightarrow X$. We obtain that $M \cong \tilde{M} / \mu \cong C(X) / \sim$ $q, \varphi$.

Hence, the structure theorem will be implied by the following: 
Theorem 2.1: Let $M$ be a compact Vaisman manifold, $L$ its weight bundle and $\Gamma \subset \mathbb{R}^{>0}$ its monodromy group. Then $\Gamma \cong \mathbb{Z}$.

For the proof, we need to look at the group generated by the Lee flow and at its lift on $\tilde{M}$. Let $\pi: \tilde{M} \longrightarrow M$ the covering associated with the monodromy group of $L$ and denote by $\operatorname{Aut}(\tilde{M}, M)$ the group of all conformal automorphisms of $\tilde{M}$ which make the following diagram commutative:

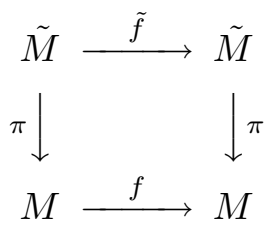

$\operatorname{Aut}(\tilde{M}, M)$ is nonempty, as it contains the Lee flow $\tilde{\Psi}_{t}$.

Let

$$
\Phi: \operatorname{Aut}(\tilde{M}, M) \longrightarrow \operatorname{Aut}(M)
$$

be the forgetful map. Recall that in (1.2) we defined the homomorphism $\rho$ mapping the group of holomorphic homotheties of $\tilde{M}$ to $\mathbb{R}^{>0}$. Restricting $\rho$ to $\operatorname{Aut}(\tilde{M}, M)$, we obtain a map $\operatorname{Aut}(\tilde{M}, M) \longrightarrow \mathbb{R}^{>0}$.

Lemma 2.2: In the above assumptions, the product map

$$
\operatorname{Aut}(\tilde{M}, M) \stackrel{\Phi \times \rho}{\longrightarrow} \operatorname{Aut}(M) \times \mathbb{R}^{>0}
$$

is injective.

Proof. Let $\tilde{f}: \tilde{M} \longrightarrow \tilde{M}$ be an automorphism such that $\Phi(\tilde{f})=i d$. Then $\tilde{f}$ is a deck transform of $\tilde{M}$. However, the group $\Gamma$ of the deck transforms of $\tilde{M}$ is identified with the monodromy of the bundle $L$ of Kähler forms on $M$. Therefore, the natural map

$$
\rho: \Gamma \longrightarrow \mathbb{R}^{>0}
$$

is injective.

Now let $G$ be the smallest Lie subgroup of $\operatorname{Aut}(M)$ containing the Lee flow $\psi_{t}$. By results in [KO], $G$ is a connected, compact abelian group of holomorphic isometries of $(M, g)$. Let $\tilde{G}=\Phi^{-1}(G)$. The main step in the proof of the structure theorem is the following:

Theorem 2.3: In the above assumptions, we have a (non-canonical) isomorphism

$$
\tilde{G} \cong \mathbb{R} \times\left(S^{1}\right)^{k}
$$

where $k=\operatorname{dim} G-1$. 
Proof. Since $G$ is a compact abelian Lie group, we have an isomorphism $G \cong$ $\left(S^{1}\right)^{k+1}$. By construction, $\tilde{G}$ is a covering of $G$. In Lemma 2.2, we constructed a natural monomorphism $\tilde{G} \longrightarrow G \times \mathbb{R}$. Denote by $\tilde{G}_{0}$ the connected component of $\tilde{G}$. Since $G$ is compact, the composition

$$
\tilde{G} \longrightarrow G \times \mathbb{R} \longrightarrow \mathbb{R}
$$

is proper. Therefore, $\tilde{G}_{0}$ is isomorphic to $\mathbb{R} \times\left(S^{1}\right)^{k}$ or to $\left(S^{1}\right)^{k+1}$. The Lee flow $\tilde{\varphi}_{t}$ multiplies the Kähler form $\omega_{k}$ by a number, hence the composition (2.3) is surjective. This implies that $\tilde{G}_{0}$ is non-compact. We obtain an isomorphism $\tilde{G}_{0} \cong \mathbb{R} \times\left(S^{1}\right)^{k}$.

Denote by $H$ the group $\tilde{G} / \tilde{G}_{0}$ of connected components of $\tilde{G}$. We first show that $H$ is finite, as follows.

Consider the restriction $\rho: \tilde{G} \longrightarrow \mathbb{R}^{>0}$, where $\rho$ is the homomorphism defined in (1.2). Let

$$
\tilde{G}_{\rho=1}:=\rho^{-1}(1)
$$

be the corresponding subgroup (formed by Kähler isometries with respect to $\omega_{k}$ ) of $\tilde{G}$. Clearly, $\rho$ is obtained by exponentiating (2.3). Since $\rho: \tilde{G} \longrightarrow \mathbb{R}$ is proper, $\tilde{G}_{\rho=1}$ is compact. To prove that $H$ is finite, we only need to show that every connected component of $\tilde{G}$ meets $\tilde{G}_{\rho=1}$.

Let $\tilde{h} \in \tilde{G}$ be an arbitrary element, $\rho(\tilde{h})=e^{t}$. By construction, $\rho\left(\tilde{\varphi}_{-t}\right)=e^{-t}$, where $\tilde{\varphi}_{t} \in \tilde{G}_{0}$ is the Lee flow of $\tilde{M}$. Then $\rho\left(\tilde{h} \cdot \tilde{\varphi}_{-t}\right)=1$, and

$$
\tilde{h} \cdot \tilde{\varphi}_{-t} \in \tilde{G}_{\rho=1} \text {. }
$$

On the other hand, $\tilde{h}$ and $\tilde{h} \cdot \tilde{\varphi}_{-t}$ belong to the same connected component of $\tilde{G}$. We obtained that every connected component of $\tilde{G}$ meets $\tilde{G}_{\rho=1}$, hence the group $H$ of connected components of $\tilde{G}$ is compact.

To finish the proof of Theorem 2.3, it remains to show that the group $H$ is trivial. By the structure theorem for abelian Lie groups, we have

$$
\tilde{G} \cong \mathbb{R} \times\left(S^{1}\right)^{k} \times H .
$$

This gives an embedding $H \hookrightarrow \tilde{G}$. Denote by $\tilde{H} \subset \tilde{G}$ its image. By definition, $\tilde{H}$ is a kernel of a projection map $\tilde{G} \longrightarrow \tilde{G}_{0}$. Since $\tilde{G}_{0}$ is mapped surjectively onto $G$, the group $\tilde{H}$ is mapped to zero under the forgetful map $\tilde{G} \longrightarrow G$. Therefore, $\tilde{H}$ is a subgroup of the monodromy group $\Gamma$. Since $\Gamma$ is the monodromy of the real vector bundle $L$, we have $\Gamma \subset \mathbb{R}$, and $\Gamma$ has no torsion subgroups. This implies that $\tilde{H}=0$. We proved Theorem 2.3.

Now the proof of the structure theorem can be completed as follows. By definition of $\tilde{G}$, the deck transformation group $\Gamma \subset \operatorname{Aut}(\tilde{M}, M)$ belongs to $\tilde{G}$. Note that $\Gamma=\operatorname{ker} \Phi$. Then, since $\tilde{G} \cong \mathbb{R} \times\left(S^{1}\right)^{k}$ and $G \cong\left(S^{1}\right)^{k+1}$, $\operatorname{ker} \Phi \cong \mathbb{Z}$. 
Remark 2.4: The proof used the hypothesis of $(M, g, J)$ being a Vaisman manifold under the equivalent statement that the Lee field has constant length and its flow consists of holomorphic isometries of the Gauduchon metric. The example of the Tricerri metric on the Inoue surface $S_{m}$ (see [Bel], [DO]) shows that harmonicity and constant length of the Lee form are not enough, even when $b_{1}=1$. Indeed, Tricerri's metric has a harmonic Lee form of (non-zero) constant length, so the fibration over $S^{1}$ exists, but the fibres are not Sasakian.

Acknowledgements : This work was finished during a visit to Ecole Polytechnique (Palaiseau). The authors thank the colleagues in the Centre de Mathématiques for warm hospitality.

\section{References}

[Bel] F.A. Belgun, On the metric structure of non-Kähler complex surfaces, Math. Ann. 317 (2000), 1-40.

[Bes] A. Besse, Einstein Manifolds, Springer-Verlag, New York, 1987.

[Bl] D.E. Blair, Riemannian geometry of contact and symplectic manifolds, Progress in Math. 203, Birkhäuser, Boston, Basel, 2002.

[BG1] C.P. Boyer, K. Galicki, 3-Sasakian Manifolds, hep-th/9810250, also published in Surveys Diff.Geom. 7 (1999) 123-184.

[DO] S. Dragomir, L. Ornea, Locally conformal Kähler geometry, Progress in Mathematics, 155. Birkhäuser, Boston, MA, 1998.

[G1] P. Gauduchon, La 1-forme de torsion d'une variete hermitienne compacte, Math. Ann., 267 (1984), 495-518.

[G2] - Structures de Weyl-Einstein, variétés de twisteurs et variétés de type $S^{1} \times S^{3}$, J. Reine Angew. Math., 469 (1995), 1-50.

[GO] P. Gauduchon, L. Ornea, Locally conformally Kähler metrics on Hopf surfaces, Ann. Inst. Fourier 48 (1998), 1107-1127.

[GOP] R. Gini, L. Ornea, M. Parton, Locally conformal Kähler reduction, math.DG/0208208, 25 pages.

[KO] Y. Kamishima, L. Ornea, Geometric flow on compact locally conformally Kähler manifolds, math.DG/0105040, 21 pages.

[MPPS] A.B. Madsen, H. Pedersen, Y.S. Poon and A. Swann, Compact Einstein-Weyl manifolds with large symmetry group, Duke Math. J. 88 (1997), 407-434.

[Sa] S. Sasaki, On differentiable manifolds with certain structures which are closely related to almost contact structure, Tohoku Math. J. 12 (1960), 459-476.

[V1] I. Vaisman, Generalized Hopf manifolds, Geom. Dedicata 13 (1982), no. 3, 231-255.

[V2] _ A survey of generalized Hopf manifolds, Rend. Sem. Mat. Torino, Special issue (1983), 205-221.

[Ve] M. Verbitsky, Vanishing theorems for locally conformal hyperkähler manifolds, 2003, math.DG/0302219, 41 pages .

University of Bucharest, Faculty of Mathematics, 14 Academiei str., 70109 BuchaREST, ROMANIA.

E-mail address: Liviu.Ornea@imar.ro

University of Glasgow, Department of Mathematics, 15 University Gardens, GlasGOW, United KingDom.

E-mail address: verbit@maths.gla.ac.uk

E-mail address: verbit@mccme.ru 